\title{
Percent Body Fat Prediction from Body Mass Index and Waist Circumference: New Cross-validated Equations for Young Adults
}

\author{
Peter D. Hart \\ Health Promotion Research, Havre, MT 59501 \\ *Corresponding author: pdhart@outlook.com
}

Received November 15, 2019; Revised December 17, 2019; Accepted December 23, 2019

\begin{abstract}
Background: A simple prediction equation that accurately predicts an individual's percent body fat (PBF) with easy to obtain inputs could benefit health and exercise science professionals. The purpose of this study was to develop and cross-validate a set of regression equations predicting PBF in young adults. Methods: A subset of $\mathrm{N}=684$ participants from a national health survey between the ages of 18 and 24 years was used in this study. Criterion values of PBF (PBF.DXA) were obtained using dual energy X-ray absorptiometry (DXA). Predictor variables included age, sex, body mass index (BMI), and waist circumference (WC). The sample was split equally and randomly into training and validation samples. Two sets of equations were evaluated in the training sample, one set using BMI and the other using WC. Both sets were tested to determine if age was a useful predictor of PBF.DXA. Cross-validation of selected model coefficients using the validation sample was evaluated using Pearson correlation coefficients, Bland and Altman limits of agreement (LOA) plots and Kappa statistics for obesity classification. Results: The selected models were both two-predictor equations: PBF.BMI2 $=10.47827+$ BMI*0.98342 12.50670; $R^{2}=.872$ and PBF.WC2 $=2.51020+\mathrm{WC} * 0.38914-13.34843 ; R^{2}=.866$. Cross-validation correlation coefficients were large for both PBF.BMI2 $(r=.91)$ and PBF.WC2 $(r=.92)$ equations. LOA plots indicated small bias of $-0.49 \pm 7.5 \%$ and $-0.25 \pm 6.8 \%$ in PBF.BMI2 and PBF.WC2 analyses, respectively. Kappa coefficients for agreement between the two obesity classification methods were considered "substantial" for PBF.BMI2 $(\kappa=.64)$ and PBF.WC2 $(\kappa=.70)$ models. Conclusion: This study provides validation evidence supporting the use of BMIand WC-based PBF prediction equations in young adult populations.
\end{abstract}

Keywords: Body composition (BC), Percent body fat (PBF), obesity, prediction equations

Cite This Article: Peter D. Hart, "Percent Body Fat Prediction from Body Mass Index and Waist Circumference: New Cross-validated Equations for Young Adults.” American Journal of Medical Sciences and Medicine, vol. 7, no. 5 (2019): 190-197. doi: 10.12691/ajmsm-7-5-2.

\section{Introduction}

The use of laboratory-based methods to measure percent body fat (PBF) can be time-consuming, expensive, and inconvenient to the participant [1,2]. Field-based PBF assessments have been developed to counter these shortcomings, however, many of them lack precision and require trained clinicians for proper administration [3]. An alternative approach is the use of a validated prediction equation utilizing a limited number of participant characteristics as inputs to estimate PBF. Several such equations exist that use body mass index (BMI) as a main predictor variable and have shown adequate validity applied to general populations $[4,5,6,7,8]$. However, not all equations generalize well to specific populations [9]. One study has shown adequate reliability for commonly used BMI-based equations predicting PBF among university students, however, predicted PBF was overestimated in both male $(+4.0 \%)$ and female $(+4.7 \%)$ students [10]. This bias may be due to the fact that college-aged individuals possess more muscle mass as compared to their older but same BMI counterparts [11]. Therefore, validation of an age-specific PBF prediction equation may be necessary. Furthermore, given research on abdominal obesity as an important predictor of health status [12,13] and the high accuracy of body circumference methods in predicting PBF [14,15], waist circumference (WC) as an input in such a prediction equation may be useful. The purpose of this study was to develop and cross-validate a set of regression equations predicting PBF that utilize BMI and WC as main input variables.

\section{Methods}

\subsection{Participants and Design}

Data for this research came from a national health survey and for this study considered a convenience sample. 
Data were collected by trained health professionals on all pertinent study variables [16]. Participants were included in the current study if they were between the ages of 18 and 24 years and had valid data for age, sex, BMI, WC and $\mathrm{PBF}$.

\subsection{Measures}

WC was assessed by a trained health professional where the measurement site was first marked on the participant's skin just above the uppermost lateral border of the right ilium at the midaxillary line [17]. A mirror was used to ensure that the steel measuring tape remained parallel to the floor. BMI was measured from participant's height and weight with weight measured on a Toledo digital scale and height measured using a stadiometer [17]. PBF was assessed using dual energy X-ray absorptiometry (DXA) [18]. DXA exams were administered by a certified radiology technologist and used the Hologic QDR 4500A. Three different scans (hip/femur, spine, and whole body) were performed on each participant, however, the whole body scan was used for determining body fat and hence was the scan used for this study. After the Hologic computer system analyzed the scans, body composition data were reported for each participant, including total PBF, hereafter referred to as PBF.DXA. Data for PBF.DXA that were either missing or invalid were subsequently imputed and as a consequence not used in this current study (discussed in the statistical analyses section). Participant age and sex were also used in this study and assessed via mobile examination center (MEC) questionnaire-based interviews [19].

\subsection{Statistical Analyses}

The first step of the analysis was to create a dataset with only complete data for all study variables. Due to the fact that imputed DXA values were reported to include extreme variability (i.e., extreme variability within the five imputed DXA values for a participant with imputed data) for some participants and due to the recommendation to treat such variability with technical analytical adjustments [20], imputed DXA data were excluded from the current study. This was achieved by first transposing the dataset, which originally included five records per participant, so that each multiple DXA record was transformed into a variable and each participant represented only a single record. At that point, imputed data were identified as values that were inconsistent across the five PBF.DXA variables. Conversely, valid measured DXA values contained a single (superfluous) value for each of the five PBF.DXA variables. Lastly, a new dummy variable was created to indicate a measured DXA participant which was used to delimit the dataset. The final dataset for this study included a single record for each participant with complete data for age, sex, BMI, WC and PBF.DXA.

Descriptive statistics were first computed for the entire sample both overall and by sex. All variable were assessed for extreme observations and influential impact using exploratory data analyses. After which, the entire sample was split equally and randomly into a training sample and validation sample. The purpose of the training sample was to build and assess competing prediction equations. A correlation matrix was developed for the training sample, which included Pearson correlation coefficients, to examine the relationship between all study variables by sex. Scatter plots were constructed with fit regression lines for each sex group to examine the predictive ability of both BMI and WC on PBF.DXA.

Four different predictive models were tested, two using BMI and two using WC. The BMI-based models were tested to determine if age was a useful predictor for PBF.DXA. This was assessed by examining the partial $F$ statistic for significant change in the coefficient of determination $\left(R^{2}\right)$ in the model including age as a predictor. The same procedure was followed for the WC-based models. Additionally, model selection criteria included comparison of $R^{2}$, standard error of estimate (SEE), and predicted residual error sum of squares (PRESS). Once the better BMI and WC models were selected, predicted values of PBF were computed using the validation sample inputs and the model coefficients from the training sample models. Descriptive statistics were computed for the validation sample which included new variables for the BMI-based predicted PBF and WCbased predicted PBF. Paired t-tests were performed to determine if significant differences were seen between each of the PBF pairs.

The selected prediction equations were cross-validated by examining: 1) Pearson correlation coefficients between criterion values of PBF and predicted values of PBF [21], 2) Bland and Altman limits of agreement (LOA) plots between the criterion and predicted PBF values [22], and 3) Cohen Kappa $(\kappa)$ coefficients for agreement between obesity classification using criterion versus predicted PBF values [23]. For Pearson correlation coefficient validation, values $>.70$ would indicate acceptable evidence [24]. For LOA plot validation, mean bias, pattern of scatter, width of limits, and percentage of values outside the limits were assessed [25]. For Kappa coefficient validation, values $\leq 0$ would indicate "no agreement", values between .01 and .20 would indicate no to "slight agreement", values between .21 and .40 would indicate "fair agreement", values between .41 and .60 would indicate "moderate agreement”, values between .61 and .80 would indicate "substantial agreement", and values $\geq .81$ would indicate almost "perfect agreement" [26]. PBF cutoff values for obese classification were $\geq 25 \%$ for male and $\geq 35 \%$ for female [27]. Additionally, each selected model's predictive accuracy was further assessed by examining the extent to which the model PRESS statistic (converted to a SE, RMPRESS) agreed with the model SEE [28]. The PRESS statistic is appropriate for cross-validation because its residuals represent the average difference between predicted values and actual values with each actual value left out of its prediction model. Therefore, the PRESS statistic attempts to remove sample dependency from the model SEE. All analyses were performed using SAS version 9.4 [29].

\section{Results}

A total of $\mathrm{N}=690$ participants had complete data for age, sex, BMI, WC and PBF.DXA. After exploratory data analyses, six observations were removed resulting in a total of $\mathrm{N}=684$ participants included in the study. Table 1 
displays descriptive statistics for the entire sample both overall and by sex. Mean BMI, WC, and PBF.DXA for male and female participants were $25.3 \mathrm{~kg} / \mathrm{m}^{2}, 86.6 \mathrm{~cm}$, $23.0 \%$ and $25.8 \mathrm{~kg} / \mathrm{m}^{2}, 86.3 \mathrm{~cm}, 36.1 \%$, respectively. After splitting the entire sample equally and randomly into training and validation samples, the training sample was used to build and assess competing prediction equations. Table 2 contains the correlation matrix for all training sample study variables by sex. As anticipated, strong correlations were found between PBF.DXA and both BMI and WC. Noteworthy was the weak correlations seen between age and PBF.DXA. This is likely due to the narrow restriction on age in this study. Additionally, the strong correlations between BMI and WC prevented their use as concurrent predictors in a single model. Figure 1 and Figure 2 display the linear relationships between PBF.DXA and both BMI and WC by sex. These graphs along with the correlation coefficients justify both BMI and WC as valid predictors of PBF.DXA.

Table 3 contains model estimates and fit statistics for the four different predictive models under consideration. Comparison of the two BMI-based models showed that age was not a useful predictor of PBF.DXA $(\Delta F=0.39$, $p=.531)$. Similar findings were seen for the two WC-based models $(\Delta F=0.73, p=.393)$. Therefore, age was not used in either of the two selected prediction equations. The two-variable BMI-based prediction equation showed large explained variance, PBF.BMI2 = 10.47827 + BMI*0.98342 - 12.50670; $R^{2}=.872$. Also, the two-variable WC-based prediction equation showed large explained variance, PBF.WC2 $=2.51020+$ WC*0.38914 $-13.34843 ; R^{2}=.866$. The above selected equations were subsequently used to predict PBF values in the validation sample using inputs from the validation sample.

Table 4 contains descriptive statistics for the training and validation samples and includes the new PBF variables (PBF.BMI2 and PBF.WC2). A significant $(p=.017)$ mean PBF difference was seen between PBF.DXA and PBF.BMI2, albeit a clinically small difference (Mean diff $=0.49 \%$ ). The mean PBF difference was however not significant $(p=.194)$ between PBF.DXA and PBF.WC2. Finally, the mean difference between the two predicted PBF estimates was significantly $(p=.031)$ different, but again a clinically small difference (Mean diff $=0.25 \%$ ). Table 5 contains cross-validation statistics between criterion values of PBF and predicted values of PBF using the newly developed prediction equations. The correlation coefficients for PBF variables were large for PBF.BMI2 $(r=.91)$ and PBF.WC2 $(r=.92)$ equations. Additionally, $R^{2}$ shrinkage was minimal for PBF.BMI2 $\left(\Delta R^{2}=.05\right)$ and PBF.WC2 $\left(\Delta R^{2}=.02\right)$ equations. Figure 3 and Figure 4 contain Bland and Altman LOA plots between the criterion and predicted PBF values. Both graphs indicate only small bias of $-0.49 \pm 7.5 \%$ and $-0.25 \pm 6.8 \%$ in PBF.BMI2 and PBF.WC2 analyses, respectively. Additionally, scatter appeared constant and random across the mean axis in both graphs, indicative of consistent measurement across the PBF range. Finally, 95\% limits were moderately wide with $4.4 \%$ and $5.8 \%$ of participants falling outside them in PBF.BMI2 and PBF.WC2 plots, respectively.

Table 1. Descriptive statistics for entire sample on prediction equation variables by sex

\begin{tabular}{|c|c|c|c|c|c|c|c|}
\hline Sample & Variable & Min & Mean & Median & $S D$ & $C V$ & $\operatorname{Max}$ \\
\hline \multirow[t]{4}{*}{ Overall $(N=684)$} & Age & 18.0 & 19.9 & 19.0 & 2.0 & 10.0 & 24.0 \\
\hline & BMI & 16.8 & 25.5 & 24.1 & 5.5 & 21.4 & 45.5 \\
\hline & WC & 56.0 & 86.4 & 83.4 & 13.9 & 16.1 & 132.9 \\
\hline & PBF.DXA & 13.0 & 29.3 & 28.9 & 9.2 & 31.5 & 52.1 \\
\hline \multirow[t]{4}{*}{ Female $(N=328)$} & Age & 18.0 & 19.9 & 19.0 & 2.0 & 9.8 & 24.0 \\
\hline & BMI & 16.8 & 25.8 & 24.0 & 6.0 & 23.4 & 45.5 \\
\hline & WC & 63.2 & 86.3 & 83.4 & 14.9 & 17.3 & 131.0 \\
\hline & PBF.DXA & 21.4 & 36.1 & 36.1 & 6.7 & 18.6 & 52.1 \\
\hline \multirow[t]{3}{*}{ Male $(N=356)$} & Age & 18.0 & 19.9 & 19.0 & 2.0 & 10.1 & 24.0 \\
\hline & WC & 56.0 & 86.6 & 83.5 & 12.9 & 14.9 & 132.9 \\
\hline & PBF.DXA & 13.0 & 23.0 & 22.0 & 6.3 & 27.4 & 40.0 \\
\hline
\end{tabular}

Note. BMI is body mass index $\left(\mathrm{kg} / \mathrm{m}^{2}\right)$. WC is waist circumference $(\mathrm{cm})$. PBF.DXA is percent body fat (PBF) by dual energy $\mathrm{X}$-ray absorptiometry (DXA). Min is minimum value. Mean is average. $S D$ is standard deviation. $C V$ is coefficient of variation. Max is maximum value.

Table 2. Correlation matrix for training sample prediction equation variables by sex

\begin{tabular}{lcccc}
\hline Variable & Age & BMI & WC & PBF.DXA \\
\hline Age & 1 & -.023 & .018 & $\mathbf{. 9 2 4}$ \\
BMI & .142 & 1 & 1 & $\mathbf{. 8 6 2}$ \\
WC &. $\mathbf{1 9 4}$ & $\mathbf{. 9 3 4}$ & $\mathbf{. 8 2 8}$ & $\mathbf{. 8 7 0}$ \\
PBF.DXA & .107 & $\mathbf{. 8 4 7}$ & 1 \\
\hline
\end{tabular}

Note. Top portion of matrix contain female $(N=175)$ coefficients and bottom portion male $(N=167)$ coefficients. Bold values are significant $(p<.05)$. 


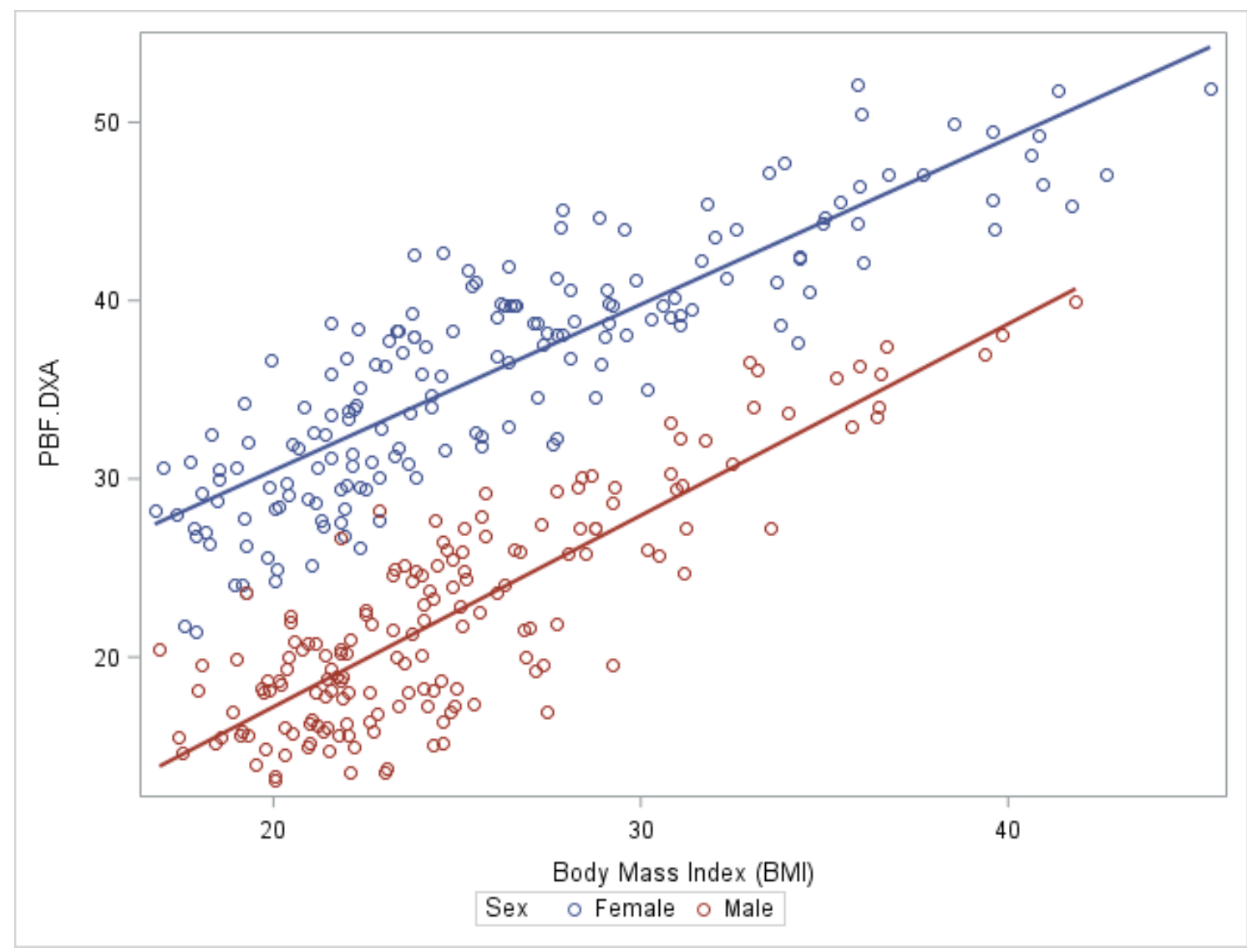

Figure 1. Scatter plot with fit regressions lines showing linear relationship between PBF.DXA and BMI by sex. (Training sample female $N=175 \&$ male $N=167$ )

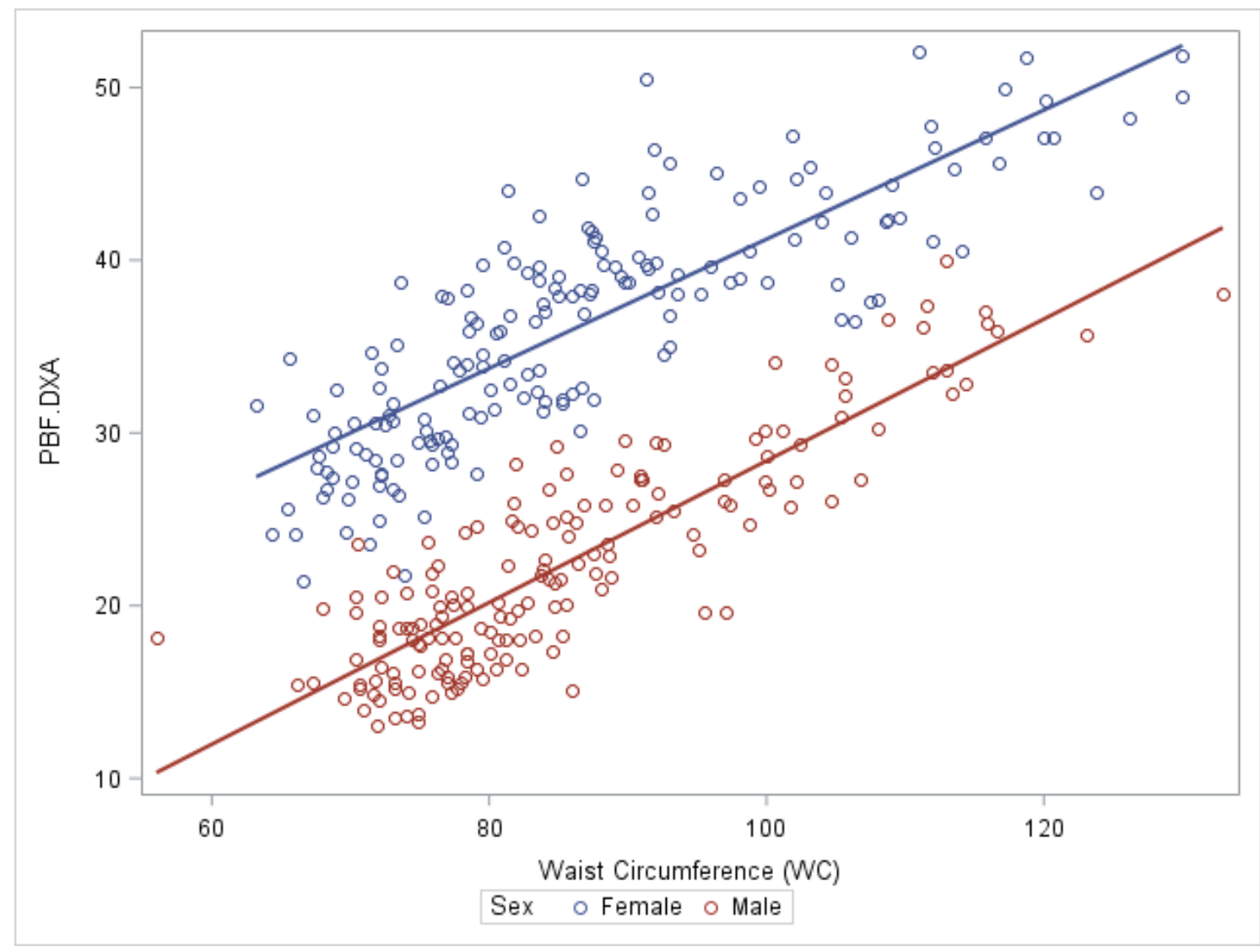

Figure 2. Scatter plot with fit regressions lines showing linear relationship between PBF.DXA and WC by sex. (Training sample female $N=175 \&$ male $N=167$ ) 
Table 3. Training sample regression models estimating criterion values of PBF (PBF.DXA) using BMI and WC as main predictor variables

\begin{tabular}{|c|c|c|c|c|c|c|c|c|c|c|c|}
\hline Model & Parameter & Estimate & $S E$ & $t$ & $p$ & VIF & $C p$ & RMPRESS & SEE & $R^{2}$ & $\Delta F$ \\
\hline \multirow[t]{4}{*}{ PBF.ВMI3 } & Intercept & 9.33197 & 2.04 & 4.58 & $<.001$ & - & 4 & 3.45 & 3.43 & .872 & 0.39 \\
\hline & BMI & 0.98243 & 0.03 & 30.04 & $<.001$ & 1.02 & & & & & \\
\hline & Sex & -12.51593 & 0.37 & -33.49 & $<.001$ & 1.02 & & & & & \\
\hline & Age & 0.05881 & 0.09 & 0.63 & .531 & 1.00 & & & & & \\
\hline \multirow[t]{3}{*}{ PBF.BMI2 } & Intercept & 10.47827 & 0.89 & 11.75 & $<.001$ & - & 3 & 3.44 & 3.43 & .872 & \\
\hline & BMI & 0.98342 & 0.03 & 30.13 & $<.001$ & 1.01 & & & & & \\
\hline & Sex & -12.50670 & 0.37 & -33.52 & $<.001$ & 1.01 & & & & & \\
\hline \multirow[t]{4}{*}{ PBF.WC3 } & Intercept & 4.05599 & 2.16 & 1.88 & .061 & - & 4 & 3.53 & 3.51 & .866 & 0.73 \\
\hline & WC & 0.39027 & 0.01 & 29.13 & $<.001$ & 1.01 & & & & & \\
\hline & Sex & -13.33595 & 0.38 & -35.11 & $<.001$ & 1.00 & & & & & \\
\hline & Age & -0.08248 & 0.10 & -0.86 & .393 & 1.01 & & & & & \\
\hline \multirow[t]{3}{*}{ PBF.WC2 } & Intercept & 2.51020 & 1.18 & 2.12 & .035 & - & 3 & 3.52 & 3.50 & .866 & \\
\hline & WC & 0.38914 & 0.01 & 29.20 & $<.001$ & 1.00 & & & & & \\
\hline & Sex & -13.34843 & 0.38 & -35.18 & $<.001$ & 1.00 & & & & & \\
\hline
\end{tabular}

Note. $N=342$. PBF.BMI3 is BMI-based prediction model with 3 predictors. PBF.BMI2 is BMI-based prediction model with 2 predictors. PBF.WC3 is WC-based prediction model with 3 predictors. PBF.WC2 is WC-based prediction model with 2 predictors. VIF is variance inflation factor where values close to 1.00 indicate no multicollinearity. $C p$ is Mallow's Cp statistic. RMPRESS is the square root of the mean predicted residual error squares. Models in bold are the suggested models. Sex is dummy coded for male $=1$.

Table 4. Descriptive statistics on prediction equation variables by random split samples

\begin{tabular}{|c|c|c|c|c|c|c|c|}
\hline Sample & Variable & Min & Mean & Median & $S D$ & $C V$ & $\operatorname{Max}$ \\
\hline \multirow[t]{4}{*}{ Training $(N=342)$} & Age & 18.0 & 20.0 & 19.0 & 2.0 & 9.9 & 24.0 \\
\hline & BMI & 16.8 & 25.5 & 24.1 & 5.7 & 22.5 & 45.5 \\
\hline & WC & 56.0 & 86.0 & 82.7 & 14.3 & 16.6 & 132.9 \\
\hline & PBF.DXA & 13.1 & 29.4 & 29.2 & 9.5 & 32.4 & 52.1 \\
\hline \multirow[t]{6}{*}{ Validation $(N=342)$} & Age & 18.0 & 19.8 & 19.0 & 2.0 & 10.0 & 24.0 \\
\hline & BMI & 16.9 & 25.6 & 24.5 & 5.2 & 20.4 & 42.5 \\
\hline & WC & 64.1 & 86.9 & 83.7 & 13.5 & 15.6 & 132.2 \\
\hline & PBF.DXA ${ }^{a, b}$ & 13.0 & 29.2 & 28.7 & 8.9 & 30.6 & 50.5 \\
\hline & PBF.BMI2 $^{a, c}$ & 14.6 & 28.7 & 29.2 & 8.0 & 27.8 & 52.3 \\
\hline & PBF.WC2 $2^{b, c}$ & 14.8 & 28.9 & 29.1 & 8.2 & 28.4 & 53.5 \\
\hline
\end{tabular}

Note. $a$ superscript indicates significant mean difference $(p=.017) . b$ superscript indicates no significant mean difference $(p=.194)$. $c$ superscript indicates significant mean difference $(p=.031)$. PBF.DXA is percent body fat (PBF) by dual energy x-ray absorptiometry (DXA). PBF.BMI2 is BMIbased prediction model with 2 predictors. PBF.WC2 is WC-based prediction model with 2 predictors.

Table 5. Cross-validation coefficients between criterion values of PBF (PBF.DXA) and predicted values of PBF

\begin{tabular}{cccccrrrrr}
\hline Equation & $r$ & $R_{Y Y^{\prime}}^{2}$ & $t$ & $p$ & & $R_{Y}^{2}$ & $\Delta R^{2}$ \\
\hline PBF.BMI2 & .905 & .819 & 39.24 & $<.001$ & & .872 & .053 \\
PBF.WC2 & .921 & .847 & 43.44 & $<.001$ & & .866 & .018 \\
\hline
\end{tabular}

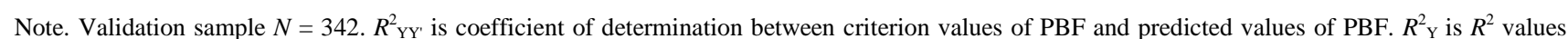
from Table 3.

Table 6 contains cross-validation statistics between criterion classification of obesity and predicted classification of obesity using the newly developed prediction equations. The kappa coefficients for agreement between the two obesity classification methods were considered "substantial" for both the PBF.BMI2 $(\kappa=.64)$ and PBF.WC2 $(\kappa=.70)$ analyses. Lastly, a final cross-validation assessment for the selected prediction equations indicated the RMPRESS statistic was similar to the SEE for both PBF.BMI2 (RMPRESS $=3.44$ vs. $S E E=3.43)$ and PBF.WC2 (RMPRESS = 3.52 vs. $S E E=3.50)$ training models. Therefore, the selected predicted equations do not appear to be sample-dependent. 


\section{LOA between PBF.DXA and PBF.BMI2}

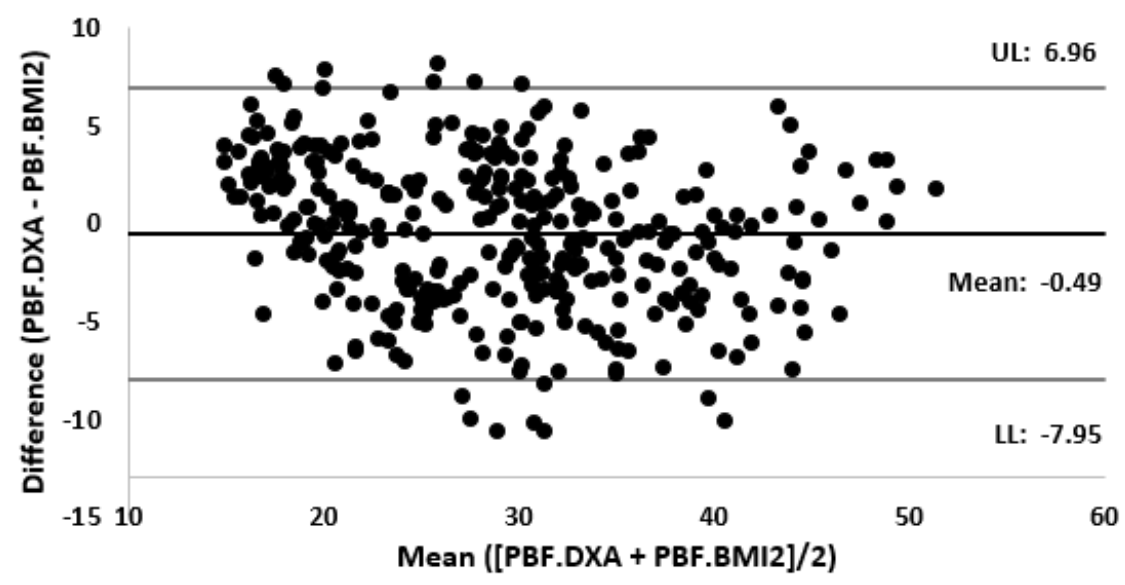

Figure 3. Bland and Altman limits of agreement (LOA) between PBF.DXA and PBF.BMI2. (Validation sample $N=342$ )

\section{LOA between PBF.DXA and PBF.WC2}

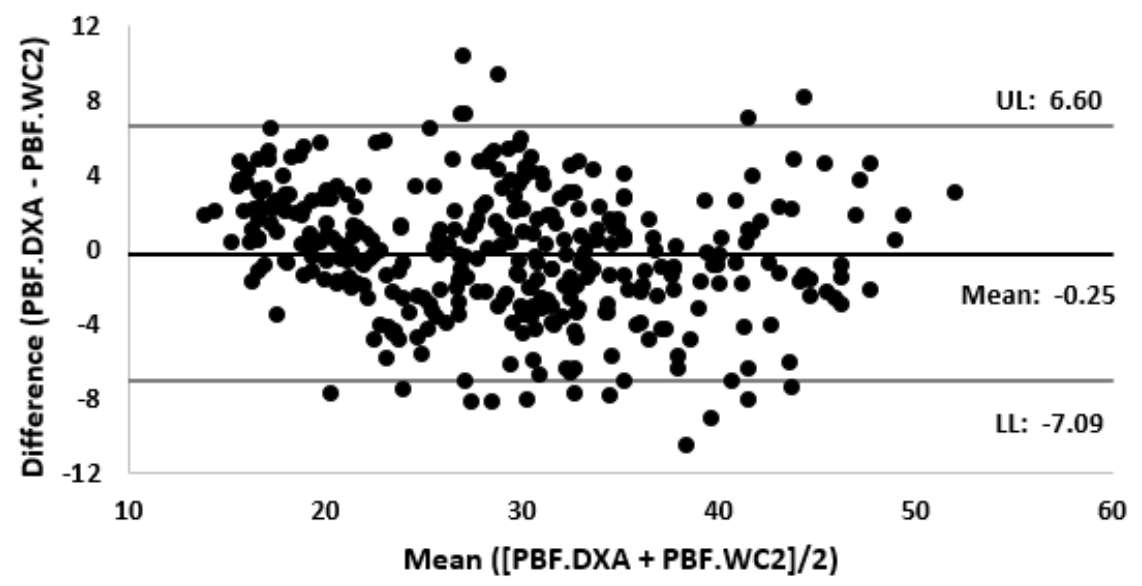

Figure 4. Bland and Altman limits of agreement (LOA) between PBF.DXA and PBF.WC2. (Validation sample $N=342$ )

Table 6. Cross-validation coefficients between criterion classification of obesity and predicted classification of obesity

\begin{tabular}{cccccc}
\hline Equation & $P$ & $\kappa$ & $L L$ & $U L$ \\
\hline PBF.BMI2 & .822 & .639 & .560 & .719 & .001 \\
PBF.WC2 & .851 & .700 & .624 & .775 \\
\hline
\end{tabular}

Note. Validation sample $N=342 . P$ is the proportion of agreement. $\kappa$ is Cohen Kappa statistic for agreement between the two obesity classifications. Cutoff values for obese classification were $\geq 25 \%$ for male and $\geq 35 \%$ for female.

\section{Discussion}

Two different prediction equations for PBF were selected for use in young adult (18 to 24 years of age) populations. The BMI-based equation (PBF.BMI2 = $\left.10.47827+\mathrm{BMI}^{*} 0.98342-12.50670\right)$ included only BMI and sex as predictors and the WC-based equation $($ PBF.WC2 $=2.51020+$ WC*0.38914 - 13.34843) included only WC and sex as predictors. Both PBF.BMI2 and PBF.WC2 models displayed acceptable internal validity. That is, 1) PBF predictors were significant contributors to their models, 2) large explained variance was seen in both models with relatively small error, 3) no excess collinearity was observed between any of the model predictors, and 4) all regression assumptions were met (i.e., PBF was linearly related to the predictor variables, errors were normally distributed with mean of zero, errors were homoscedastic and independent across predictor variables). Additionally, PBF.BMI2 and PBF.WC2 models showed acceptable external validity. More specifically, 1) correlations were strong between criterion values of PBF and cross-validated predicted values of PBF, 2) minimal shrinkage was observed using the training model coefficients on the validation samples, 3) no troubling bias was seen when examining the differences between the criterion and predicted values of PBF, 4) acceptable agreement was noted between obesity classification using criterion and predicted values of PBF, and 5) PBF predictions were not dependent on the training sample as noticed by the close proximity of the model errors when a leave-one-out procedure was conducted.

Many strengths are worth mentioning regarding the development of both PBF.BMI2 and PBF.WC2 equations. One strength is that a large national sample was used in their construction. Although this sample was not representative of all U.S. young adults in this age group, 
due to exclusion of imputed DXA data and no adjustments made to the standard errors for complex sampling, the sample was still drawn from a relatively large pool of U.S. citizens. A second strength that adds to the credibility of these equations is the use of DXA in the measurement of PBF. DXA, which is considered a gold-standard method for such assessments [30]. A third and last strength worth mentioning is the use of trained health clinicians in the measurement of both BMI and WC, the main predictor variables for these equations. Therefore, these equations were developed using reliable measurements and not self-reported values obtained from participants.

Some cautionary notes are needed however before one considers using either PBF.BMI2 or PBF.WC2 in young adult populations. One important consideration is the generalization of PBF.BMI2 and PBF.WC2 to similar populations. It is known that two general types of prediction equations exist: generalized and population specific [31]. However, in viewing the population-specific characteristics of this study's equations, they are still likely generalized equations within the age range of 18 to 24 years. That is, it is not known if equations PBF.BMI2 and PBF.WC2 generalize to college students, athletes, or highly trained young adults. Therefore, these equations should be considered to represent valid predictions for the general young adult 18 to 24 years of age population. A final consideration regarding the use of PBF.BMI2 and PBF.WC2 is their superior accuracy in norm-referenced over criterion-referenced evaluation. Specifically, the correlations found in the cross-validation part of this study were considered strong validity evidence for the prediction equations. However, Pearson correlation coefficients are only useful for assessing the ability of scores to rank individuals on some trait of interest [32]. If however, interest is on measuring an individual to obtain an exact value, then criterion-referenced methods are required. Since this study found a relatively moderate spread around the mean difference of criterion and predicted PBF and also failed to find "perfect" agreement between the two obesity classification methods, this latter form of assessment should be considered weaker than the former.

\section{Conclusions}

Two PBF prediction equations were developed and cross-validated on a sample population of young adults. These equations are BMI-based (PBF.BMI2 = $10.47827+$ BMI*0.98342 - 12.50670) and WC-based (PBF.WC2 = $2.51020+$ WC*0.38914 - 13.34843). Both equations met cross-validation criteria with the WC-based model providing marginally superior predictive properties. Health and fitness professionals should consider using these equations because of their accuracy and easy to obtain inputs. These equations, however, may not generalize well in highly trained populations.

\section{Acknowledgements}

No financial assistance was used to assist with this project.

\section{References}

[1] Henry CJ, Shalini D, Ponnalagu O, Bi X, Tan SY. New equations to predict body fat in Asian-Chinese adults using age, height, skinfold thickness, and waist circumference. Journal of the Academy of Nutrition and Dietetics. 2018 Jul 1; 118(7): 1263-9.

[2] Hendrickson N, Davison J, Schiller L, Willey M. Reliability and Validity of A-Mode Ultrasound to Quantify Body Composition. Journal of orthopaedic trauma. 2019 Sep 1; 33(9): 472-7.

[3] Loenneke JP, Barnes JT, Wilson JM, Lowery RP, Isaacs MN, Pujol TJ. Reliability of field methods for estimating body fat. Clinical physiology and functional imaging. 2013 Sep; 33(5): 405-8.

[4] Deurenberg, P., Weststrate, J.A. and Seidell, J.C., 1991. Body mass index as a measure of body fatness: age-and sex-specific prediction formulas. British journal of nutrition, 65(2), pp.105-114

[5] Deurenberg, P., Yap, M. and Van Staveren, W.A., 1998. Body mass index and percent body fat: a meta-analysis among different ethnic groups. International journal of obesity, 22(12), p.1164.

[6] Gallagher, D., Visser, M., Sepulveda, D., Pierson, R.N., Harris, T. and Heymsfield, S.B., 1996. How useful is body mass index for comparison of body fatness across age, sex, and ethnic groups?. American journal of epidemiology, 143(3), pp.228-239.

[7] Jackson, A.S., Pollock, M.L. and Ward, A.N.N., 1980. Generalized equations for predicting body density of women. Medicine and science in sports and exercise, 12(3), pp.175-181.

[8] Jackson, A.S., Stanforth, P.R., Gagnon, J., Rankinen, T., Leon, A.S., Rao, D.C., Skinner, J.S., Bouchard, C. and Wilmore, J.H., 2002. The effect of sex, age and race on estimating percentage body fat from body mass index: The Heritage Family Study. International journal of obesity, 26(6), p.789.

[9] Veronesi G, Gianfagna F, Giampaoli S, Chambless LE, Grassi G, Cesana G, Ferrario MM. Validity of a long-term cardiovascular disease risk prediction equation for low-incidence populations: The CAMUNI-MATISS Cohorts Collaboration Study. European journal of preventive cardiology. 2015 Dec; 22(12): 1618-25.

[10] Hart PD. Equivalence Reliability and Convergent Validity of Percent Body Fat Prediction Equations. American Journal of Sports Science and Medicine. 2019; 7(2): 45-50.

[11] Siparsky PN, Kirkendall DT, Garrett Jr WE. Muscle changes in aging: understanding sarcopenia. Sports Health. 2014 Jan; 6(1): 36-40.

[12] Hart PD. Self-reported physical inactivity and waist circumference independently predict all-cause mortality in US adults. American Journal of Public Health Research. 2017;5(6):184-9.

[13] Hart PD. Concurrent relationship of objectively measured physical activity and cardiorespiratory fitness on two different measures of obesity in U.S. adults. Journal of Physical Activity Research. 2018; 3(2): 78-81.

[14] Hodgdon JA, Beckett MB. Prediction of percent body fat for US Navy women from body circumferences and height. Naval Health Research Center San Diego Ca; Report No. 84-29. 1984.

[15] Hodgdon JA, Beckett MB. Prediction of percent body fat for US Navy men from body circumferences and height. Naval Health Research Center San Diego Ca; Report No. 84-11. 1984.

[16] Zipf G, Chiappa M, Porter KS, et al. National Health and Nutrition Examination Survey: Plan and operations, 1999-2010. National Center for Health Statistics. Vital Health Stat 1(56). 2013.

[17] Centers for Disease Control and Prevention National Center for Health Statistics. NHANES 2005-2006 Anthropometry and Physical Activity Monitor Procedures Manual; 2005.

[18] Centers for Disease Control and Prevention National Center for Health Statistics. NHANES 2005-2006 Body Composition Procedures Manual; 2006.

[19] Centers for Disease Control and Prevention National Center for Health Statistics. National Health and Nutrition Examination Survey (NHANES): MEC Interviewers Procedures Manual; 2006.

[20] Centers for Disease Control and Prevention National Center for Health Statistics. National Health and Nutrition Examination Survey: Technical Documentation for the 1999-2004 Dual Energy X-Ray Absorptiometry (DXA) Multiple Imputation Data files; 2006.

[21] Tabachnick BG, Fidell LS, Ullman JB. Using multivariate statistics. Boston, MA: Pearson; 2013. 
[22] Bland, J.M. and Altman, D., 1986. Statistical methods for assessing agreement between two methods of clinical measurement. The lancet, 327(8476), pp.307-310.

[23] Howell DC. Statistical methods for psychology. Cengage Learning; 2012.

[24] Carlson, K.D. and Herdman, A.O., 2012. Understanding the impact of convergent validity on research results. Organizational Research Methods, 15(1), pp.17-32.

[25] Giavarina D. Understanding Bland Altman analysis. Biochemia medica: Biochemia medica. 2015 Jun 15; 25(2): 141-51.

[26] Landis, J.R. and Koch, G.G., 1977. The measurement of observer agreement for categorical data. biometrics, pp.159-174.

[27] Heinrich KM, Jitnarin N, Suminski RR, Berkel L, Hunter CM, Alvarez L, Brundige AR, Peterson AL, Foreyt JP, Haddock CK, Poston WS. Obesity classification in military personnel: a comparison of body fat, waist circumference, and body mass index measurements. Military medicine. 2008 Jan 1; 173(1): 67-73.

[28] Neter J. W, Wasserman, and MH Kutner. 1990. Applied linear statistical models: regression, analysis of variance, and experimental designs, 3rd ed. RD Irwin Press, Homewood, Illinois.

[29] Cody, R. and Smith, J. Applied statistics and the SAS programming language. Pearson; 5th edition; 2005

[30] Kenney, W.L., Wilmore, J.H. and Costill, D.L., 2015. Physiology of sport and exercise. Human kinetics.

[31] Gibson AL, Wagner D, Heyward V. Advanced Fitness Assessment and Exercise Prescription, 8E. Human kinetics; 2018 Sep 27.

[32] Morrow Jr JR, Mood D, Disch J, Kang M. Measurement and Evaluation in Human Performance, 5E. Human Kinetics; 2015 Aug 25.

(C) The Author(s) 2019. This article is an open access article distributed under the terms and conditions of the Creative Commons Attribution (CC BY) license (http://creativecommons.org/licenses/by/4.0/). 